EPJ Web of Conferences 19, 09009 (2012)

DOI: $10.1051 /$ epjconf/20121909009

(C) Owned by the authors, published by EDP Sciences, 2012

\title{
VVV: The near-IR Milky Way bulge and plane survey*
}

\author{
M. Rejkuba ${ }^{1, a}$, O.A. Gonzalez ${ }^{1}$, I. Toledo² ${ }^{2}$ D. Minniti'3,4,5, P. Lucas ${ }^{6}$ \\ and the VVV team
}

${ }^{1}$ ESO, Karl-Schwarzschild-Strasse 2, 85748 Garching b. München, Germany

${ }^{2}$ Atacama Large Millimeter Array, Alonso de Córdova 3107, Vitacura, Santiago, Chile

${ }^{3}$ Dept. Astronomía y Astrofísica, Pontificia Univ. Católica de Chile,

Av. Vicuña Mackenna 4860, Santiago, Chile

${ }^{4}$ Vatican Observatory, Vatican City State V-00120, Italy

${ }^{5}$ Dept. of Astrophysical Sciences, Princeton University, Princeton, NJ 08544-1001, USA

${ }^{6}$ Centre for Astrophysics Research, Univ. of Hertfordshire, College Lane, Hatfield AL10 9AB,

UK

\begin{abstract}
The ESO public survey "VISTA Variables in the Via Lactea" (VVV) started mapping the inner disk and bulge of our Galaxy with the VISTA 4m telescope in the near-IR in 2010. The planned survey area of $520 \mathrm{deg}^{2}$ is observed in the Z, Y, J, H and Ks filters, and in addition more than 100 epochs of repeated imaging in Ks will be collected over $\sim 5$ years. The final products will be a deep near-IR atlas in five passbands, and catalogue of more than a million variable sources. This public survey will provide data available to the whole community and therefore will enable further studies of the history of the Milky Way, its star cluster evolution, and the population census of the Galactic Bulge and center, as well as the investigations of the star formation regions in the disk.
\end{abstract}

\section{VVV SURVEY MAIN PARAMETERS}

The VVV public survey area covers $\sim 220 \mathrm{sq}$. deg in the inner disk within the following Galactic coordinate limits: $-2^{\circ}<b<+2^{\circ}$ and $-65^{\circ}<l<-10^{\circ}$. In the bulge the survey area is $\sim 300 \mathrm{sq}$. deg and covers $-10^{\circ}<b<+5^{\circ}$ and $-10^{\circ}<l<+10^{\circ}$. This area is divided into 152 fields in the disk, and 196 in the bulge, each $1 \times 1.5$ sq. deg in size.

The observing strategy includes a full pass of the survey area in year 1 (2010) in all five filters: Z, Y, $\mathrm{J}, \mathrm{H}$, and Ks, spanning 0.9-2.5 $\mu \mathrm{m}$, to magnitude limits of 20.5, 20, 20, 19 and $18 \mathrm{mag}$, respectively. The main variability campaign for the bulge part of the survey is scheduled in 2012 and for the disk in 2013, but the first multi-epoch observations in Ks-band have already started in 2010. By the end of the survey we expect to have up to 100 epochs in all fields, which will enable in-depth study of variable sources, most of which will be RR Lyrae in the bulge, and Cepheids in the disk fields. Using these well understood distance indicators it will be possible to derive 3-D map of the surveyed region, essentially giving one extra dimension to VVV with respect to other surveys such as 2MASS.

The VVV photometric limit is about four magnitudes deeper than 2MASS in J, H and Ks filters and it adds two more bands. In addition to the depth of the survey, another notable advantage over other

\footnotetext{
*Based on Observations Collected within ESO Public Survey VVV: 179.B-2002.

ae-mail: mrejkuba@eso.org
}

This is an Open Access article distributed under the terms of the Creative Commons Attribution-Noncommercial License 3.0, which permits unrestricted use, distribution, and reproduction in any noncommercial medium, provided the original work is properly cited. 


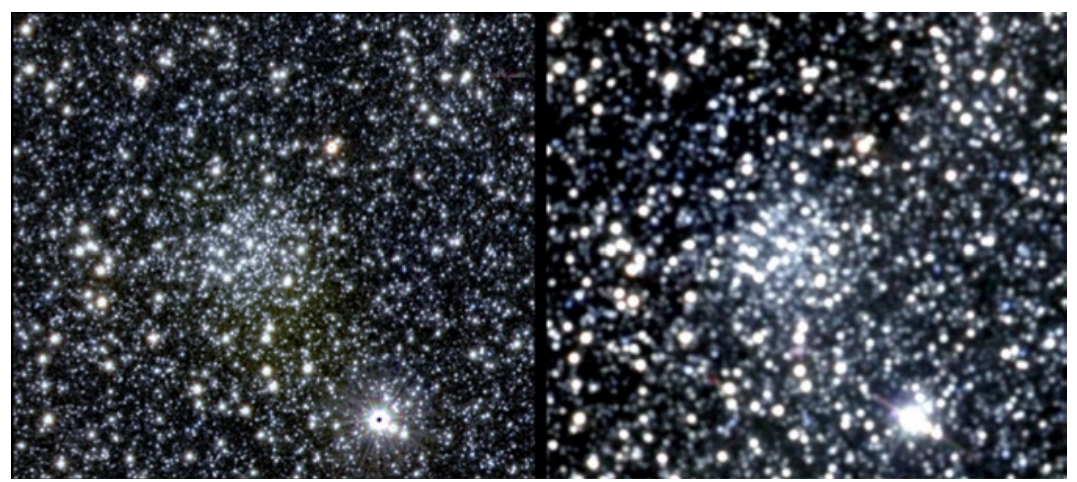

Figure 1. Comparison between VVV (left) and 2MASS (right) for a small area centered on globular cluster Pal 6.

available data is the high spatial resolution of images (Fig. 1). The camera has $0.34^{\prime \prime}$ pixels, and the median image quality is $0.9^{\prime \prime}$ in JHK, and $1.0^{\prime \prime}$ in $\mathrm{Y}$ and $\mathrm{Z}$ images, as measured on the data.

For a more detailed description of the survey goals and observing strategy we refer to Minniti et al. [5] and the survey web page: http://vvvsurvey.org.

\section{VVV FIRST YEAR PROGRESS}

In the first observing season (2010) all disk fields and 92\% of bulge fields were completed in JHKs, while YZ completion is $84 \%$ for disk and $40 \%$ for the bulge areas. Since then, in 2011 , the whole area has been covered with single-epoch multi-band observations.

The data are processed through the VISTA Data Flow System (VDFS), and after verification by the team the first public data release has been published in July 2011 through the ESO archive ${ }^{1}$. This first year data release, version 1.0, contains reduced tile images and associated source lists for all observations completed until October 2010. As the observations are advancing, the pipeline is also being improved. The future releases will contain more accurate calibrations as well as more advanced data products, such as multi-band catalogues and light-curves for variable sources. The plan is to make incremental public data releases on a yearly basis.

\section{FIRST RESULTS AND DISCOVERY POTENTIAL}

Papers by Saito et al. [8] and Catelan et al. [2] show the potential of the VVV survey for the study of star clusters, stellar populations, extinction, variable star properties, star formation, planetary nebulae, post-AGB stars, as well as for the study of much closer targets, such as asteroids, near-Earth objects, and TNOs. Already during the first year many new open and globular cluster candidates were discovered $[1,6,7]$, in only a subset of the whole area. The high resolution and uniform coverage of the whole bulge in 5 broad-band filters will enable construction of homogeneous and high resolution extinction and metallicity distribution maps of the bulge [3], which can then be used to study in detail the structure of the inner and outer bulge [4, 9].

\footnotetext{
${ }^{1}$ http://archive.eso.org/wdb/wdb/adp/phase3_vircam/form
} 
Assembling the Puzzle of the Milky Way

\section{References}

[1] Borissova, J., et al., 2011, A\&A, 532, A131

[2] Catelan, M., et al., 2011, Carnegie Obs. Astrophysics Series, V. 5, arXiv:1105.1119

[3] Gonzalez, O. A., Rejkuba, M., Zoccali, M., et al., 2011a, A\&A, 534, A3

[4] Gonzalez, O. A., Rejkuba, M., Minniti, D., et al., 2011b, A\&ALett, 534, L14

[5] Minniti, D., et al., 2010, New Astronomy, 15, 433

[6] Minniti, D., Hempel, M., Toledo, I., et al., 2011, A\&A, 527, A81

[7] Moni Bidin, C., et al., 2011, A\&A, 535, A33

[8] Saito R., et al., 2010, The Messenger, 141, 24

[9] Saito, R., Zoccali, M., McWilliam, A., et al., 2011, AJ, 142, 76 